\title{
ON THE REMAINDER OF GAUSSIAN QUADRATURE FORMULAS FOR BERNSTEIN-SZEGÖ WEIGHT FUNCTIONS
}

\author{
F. PEHERSTORFER
}

\begin{abstract}
We give an explicit expression for the kernel of the error functional for Gaussian quadrature formulas with respect to weight functions of BernsteinSzegö type, i.e., weight functions of the form $(1-x)^{\alpha}(1+x)^{\beta} / \rho(x), x \in$ $(-1,1)$, where $\alpha, \beta \in\left\{-\frac{1}{2}, \frac{1}{2}\right\}$ and $\rho$ is a polynomial of arbitrary degree which is positive on $[-1,1]$. With the help of this result the norm of the error functional can easily be calculated explicitly for a wide subclass of these weight functions.
\end{abstract}

\section{INTRODUCTION AND NOTATION}

We consider Gaussian quadrature formulas with respect to a nonnegative weight function $w$ on the interval $[-1,1]$,

$$
\int_{-1}^{1} f(x) w(x) d x=\sum_{j=1}^{n} \lambda_{j} f\left(x_{j}\right)+R_{n}(f, w),
$$

where $x_{j}=x_{j, n}$ are the zeros of the $n$ th-degree monic orthogonal polynomial $P_{n}(\cdot, w)$ and $\lambda_{j}=\lambda_{j, n}$ are the corresponding Christoffel numbers. If $f$ is analytic in a domain $D$ which contains in its interior the interval $[-1,1]$ and a contour $\Gamma$ surrounding $[-1,1]$, the remainder term can be represented as a contour integral (see, e.g., [3])

$$
R_{n}(f, w)=\frac{1}{2 \pi i} \int_{\Gamma} K_{n}(z, w) f(z) d z,
$$

where the kernel $K_{n}(\cdot, w)$ is given by

$$
K_{n}(z, w)=R_{n}\left(\frac{1}{z-\cdot}, w\right)
$$

or, alternatively, by

$$
K_{n}(z, w)=\frac{Q_{n}(z, w)}{P_{n}(z, w)},
$$

where $Q_{n}(\cdot, w)$ is the $n$th function of the second kind, i.e.,

$$
Q_{n}(z, w)=\int_{-1}^{1} \frac{P_{n}(x, w)}{z-x} w(x) d x \text { for } z \in \mathbf{C} \backslash[-1,1] .
$$

Received by the editor October 30,1990 and, in revised form, November 5, 1991 and February 10, 1992.

1991 Mathematics Subject Classification. Primary 65D32; Secondary 33C45. 
Let us note that by $(1.3), K_{n}(\cdot, w)$ has the following series expansion:

$$
K_{n}(z, w)=\sum_{k=2 n}^{\infty} \frac{R_{n}\left(x^{k}, w\right)}{z^{k+1}} \text { for }|z|>1 .
$$

From (1.2) the following well-known estimate of the remainder, based on contour integration, follows:

$$
\left|R_{n}(f, w)\right| \leq \frac{l(\Gamma)}{2 \pi} \max _{z \in \Gamma}\left|K_{n}(z, w)\right| \max _{z \in \Gamma}|f(z)|,
$$

where $l(\Gamma)$ denotes the length of $\Gamma$.

Another useful method to estimate the remainder for a function analytic in $C_{r}=\{z \in \mathbf{C}:|z|<r\}, r>1$, has been suggested by Hämmerlin [4], namely: For a function $f(z)=\sum_{k=0}^{\infty} a_{k}(f) z^{k}$ analytic in $C_{r}$ define

$$
|f|_{r}:=\sup \left\{\left|a_{k}(f)\right| r^{k}: k \in \mathbf{N}_{0} \text { and } R_{n}\left(x^{k}, w\right) \neq 0\right\} \text {. }
$$

Then, $|\cdot|_{r}$ in the space

$$
\mathfrak{X}_{r}:=\left\{f: f \text { analytic in } C_{r} \text { and }|f|_{r}<\infty\right\}
$$

is a seminorm. The error functional $R_{n}(f, w)$ is continuous in $\left(\mathfrak{X}_{r},|\cdot|_{r}\right)$, and we have

$$
\left|R_{n}(f, w)\right| \leq\left\|R_{n}\right\||f|_{r},
$$

where $\left\|R_{n}\right\|$ can be estimated by

$$
\left\|R_{n}\right\| \leq \sum_{k=2 n}^{\infty} \frac{\left|R_{n}\left(x^{k}, w\right)\right|}{r^{k}} .
$$

Equality holds (put $f(z)=1 /(r-z)$, resp. $1 /(r+z)$ ) if for all $k \geq 2 n$ the condition

$$
R_{n}\left(x^{k}, w\right) \geq 0, \quad \operatorname{resp} .(-1)^{k} R_{n}\left(x^{k}, w\right) \geq 0,
$$

is fulfilled. Since by [3, Theorem 2.1], proved in [2], and the proof of Theorem 3.1 in [3], the condition

$$
w(x) / w(-x) \text { nondecreasing on }(-1,1),
$$

resp.

$$
w(x) / w(-x) \text { nonincreasing on }(-1,1),
$$

implies that condition (1.9) holds for all $k \in \mathbf{N}_{0}$, it follows by (1.6) (see [3, Theorem 3.1]) that

$$
\max _{|z|=r}\left|K_{n}(z, w)\right|= \begin{cases}K_{n}(r, w) & \text { if } w \text { satisfies }(1.10), \\ \left|K_{n}(-r, w)\right| & \text { if } w \text { satisfies }(1.11)\end{cases}
$$

and

$$
\left\|R_{n}\right\|= \begin{cases}r K_{n}(r, w) & \text { if } w \text { satisfies (1.10), } \\ -r K_{n}(-r, w) & \text { if } w \text { satisfies (1.11). }\end{cases}
$$

Thus, we see that for the estimation of the remainder it is very desirable to have an explicit expression for the kernel $K_{n}(z, w)$. 
Very recently, Notaris [8] computed $\left\|R_{n}\right\|$ explicitly for weight functions of the form

$$
w(x)=(1-x)^{\alpha}(1+x)^{\beta} / \rho_{2}(x) \text { for } x \in(-1,1),
$$

where $\alpha, \beta \in\left\{-\frac{1}{2}, \frac{1}{2}\right\}$ and $\rho_{2}$ is a polynomial of degree at most two which is positive on $[-1,1]$ and satisfies condition (1.10) or (1.11). For the special case when $\rho_{2}$ is a polynomial of degree one or a particular even polynomial of degree two, this has been done before by Akrivis [1] (see also Kumar [5, 6]). Let us also mention that a detailed study of the kernel function for the four Chebyshev weight functions, i.e., $\rho_{2}(x) \equiv 1$ in (1.12), can be found in Gautschi and Varga [3]. In this note we derive an explicit expression for the kernel $K_{n}(z, w)\left(\left\|R_{n}\right\|\right)$ for all Bernstein-Szegö weight functions $w$ (which satisfy condition (1.10) or (1.11)), where a weight function is called a Bernstein-Szegö weight function if it is of the form

$$
\pi w_{\alpha, \beta}\left(x, \rho_{m}\right)=(1-x)^{\alpha}(1+x)^{\beta} / \rho_{m}(x) \text { for } x \in(-1,1),
$$

with $\alpha, \beta \in\left\{-\frac{1}{2}, \frac{1}{2}\right\}$ and $\rho_{m}$ a polynomial of degree $m, m$ arbitrary, which is positive on $[-1,1]$.

\section{MAIN RESUlt}

First let us recall the well-known fact that the so-called Joukowski transformation

$$
y=\frac{1}{2}\left(z+z^{-1}\right)
$$

maps $\{z \in \mathbf{C}:|z|<1\} \backslash\{0\} \quad(\{z \in \mathbf{C}:|z|>1\})$ one-to-one onto $\mathbf{C} \backslash[-1,1]$ and that the inverse transformation is given by

$$
z=y_{(+)}^{-} \sqrt{y^{2}-1}
$$

where that branch of $\sqrt{ }$ is chosen for which $\sqrt{y^{2}-1}>0$ for $y \in(1, \infty)$. Note that the transformation (2.1) maps the circumference $|z|=1$ onto the interval $[-1,1]$.

The following version of the Fejér-Riesz Theorem on the representation of positive trigonometric polynomials (compare Theorems 1.2.1 and 1.2.2 in [10]) will be needed.

Lemma. Let $\rho_{m}$ be a real positive polynomial on $[-1,1]$ of exact degree $m$. Then there exists a unique real polynomial

$$
g_{m}(z)=\prod_{\nu=1}^{m}\left(z-z_{\nu}\right) \quad \text { with } 0<\left|z_{\nu}\right|<1 \text { for } \nu=1, \ldots, m
$$

such that

$$
\rho_{m}(\cos \varphi)=c\left|g_{m}\left(e^{i \varphi}\right)\right|^{2} \quad \text { for } \varphi \in[0,2 \pi],
$$

where $c \in \mathbf{R}^{+}$.

Proof. Let $\rho_{m}(x)=\tilde{c} \prod_{\nu=1}^{m}\left(\alpha_{\nu}-x\right)$, where $\tilde{c} \in \mathbf{R}$ and the $\alpha_{\nu}$ 's are either in $\mathbf{R} \backslash[-1,1]$ or appear in pairs of complex conjugate numbers. Hence, if we set

$$
z_{\nu}=\alpha_{\nu}-\sqrt{\alpha_{\nu}^{2}-1} \text { for } \nu=1, \ldots, m,
$$


then

$$
0<\left|z_{\nu}\right|<1 \text { and } \alpha_{\nu}=\frac{1}{2}\left(z_{\nu}+z_{\nu}^{-1}\right) \text { for } \nu=1, \ldots, m
$$

Thus,

since

$$
\rho_{m}(\cos \varphi)=\tilde{c} \prod_{\nu=1}^{m}\left(\frac{1+z_{\nu}^{2}}{2 z_{\nu}}-\cos \varphi\right)=c \prod_{\nu=1}^{m}\left|e^{i \varphi}-z_{\nu}\right|^{2}
$$

$$
\left(e^{i \varphi}-z_{\nu}\right)\left(e^{-i \varphi}-z_{\nu}\right)=2 z_{\nu}\left(\frac{1+z_{\nu}^{2}}{2 z_{\nu}}-\cos \varphi\right)
$$

and the $z_{\nu}$ 's are real or appear in pairs of complex conjugate numbers.

Now the uniqueness remains to be shown. Suppose that

$$
\rho_{m}(\cos \varphi)=d \prod_{\nu=1}^{m}\left|e^{i \varphi}-v_{\nu}\right|^{2}
$$

where $d \in \mathbf{R}^{+}, v_{\nu} \in\{z \in \mathbf{C}:|z|<1\} \backslash\{0\}$ for $\nu=1, \ldots, m$, and the $v_{\nu}$ 's are real or complex conjugate. Then it follows as above that $\left(v_{\nu}+v_{\nu}^{-1}\right) / 2$, $\nu=1, \ldots, m$, is a zero of $\rho_{m}(\cos \varphi)$ and thus, since $0<\left|v_{\nu}\right|<1$ and since the Joukowski transformation is one-to-one, the uniqueness follows.

Let us note that other representations of $\rho_{m}$ of the form (2.4), but with $g_{m}$ having $m-l$, resp. $l, l \in\{1, \ldots, m\}$, zeros inside, resp. outside, of the unit disk, can be obtained by replacing $(2.5)$ by

$$
z_{\nu_{j}}=\alpha_{\nu_{j}}+\sqrt{\alpha_{\nu_{j}}^{2}-1} \text { for } j=1, \ldots, l,
$$

where $\left\{\nu_{1}, \ldots, \nu_{l}\right\}$ is an arbitrary subset of $\{1, \ldots, m\}$, and

$$
z_{\nu}=\alpha_{\nu}-\sqrt{\alpha_{\nu}^{2}-1} \text { for } \nu \in\{1, \ldots, m\} \backslash\left\{\nu_{1}, \ldots, \nu_{l}\right\}
$$

Now let us set

$$
\pi w\left(x, \rho_{m}\right)=1 /\left(\sqrt{1-x^{2}} \rho_{m}(x)\right) \text { for } x \in(-1,1)
$$

and let $g_{m}$ be the unique polynomial from the above lemma. Then it follows by well-known results of Bernstein and Szegö (see, e.g., [10, p. 31] and set $h_{m}(z)=\sqrt{c \pi} z^{m} g_{m}\left(\frac{1}{z}\right)$ there) that, with $z=e^{i \varphi}$ and $x=\cos \varphi$,

$$
\begin{aligned}
& 2^{n-1} P_{n}\left(x, w\left(\cdot, \rho_{m}\right)\right)=\sum_{j=0}^{m} a_{j} T_{n-j}(x) \\
& \quad=\operatorname{Re}\left\{z^{n-m} g_{m}(z)\right\} \text { for } 2 n>m, \\
& 2^{n-1} P_{n-1}\left(x,\left(1-x^{2}\right) w\left(\cdot, \rho_{m}\right)\right)=\sum_{j=0}^{m} a_{j} U_{n-1-j}(x) \\
& \quad=\operatorname{Im}\left\{z^{n-m} g_{m}(z)\right\} / \sin \varphi \text { for } 2 n>m, \\
& 2^{n} P_{n}\left(x,(1+x) w\left(\cdot, \rho_{m}\right)\right)=\sum_{j=0}^{m} a_{j} \frac{T_{n+1-j}(x)+T_{n-j}(x)}{x+1} \\
& \quad=\operatorname{Re}\left\{z^{n-m+1 / 2} g_{m}(z)\right\} / \cos (\varphi / 2) \text { for } 2 n+1>m, \\
& 2^{n} P_{n}\left(x,(1-x) w\left(\cdot, \rho_{m}\right)\right)=\sum_{j=0}^{m} a_{j} \frac{T_{n+1-j}(x)-T_{n-j}(x)}{x-1} \\
& \quad=\operatorname{Im}\left\{z^{n-m+1 / 2} g_{m}(z)\right\} / \sin (\varphi / 2) \text { for } 2 n+1>m,
\end{aligned}
$$


where $g_{m}(z)=\sum_{j=0}^{m} a_{j} z^{m-j}$ and $T_{j}$, resp. $U_{j}$, denotes the Chebyshev polynomial of degree $j$ of the first, resp. second, kind on $[-1,1]$.

We mention in passing that if $g_{m}$ in (2.7) is replaced by a polynomial $\tilde{g}_{m}$ which also satisfies (2.4) but does not have all zeros in the open unit disk, then the polynomials on the right-hand side in (2.7) are not orthogonal with respect to $w_{\alpha, \beta}\left(\cdot, \rho_{m}\right), \alpha, \beta \in\left\{-\frac{1}{2}, \frac{1}{2}\right\}$. In fact (see [9, Corollary 5], corresponding results hold also for $\alpha=-\beta= \pm \frac{1}{2}$ ), they are orthogonal with respect to a functional $\Psi$ of the form

$$
\Psi(p)=\int_{-1}^{+1} p(x) w_{\alpha, \beta}\left(x, \rho_{m}\right) d x+L(p) \text { for } p \in \mathbf{P},
$$

where $L$ is a functional given by

$$
L(p)=\sum_{j=1}^{l^{*}} \sum_{\kappa=1}^{l_{j}} \mu_{\kappa, j} p^{(\kappa-1)}\left(\alpha_{\nu_{j}}\right)
$$

and the $\alpha_{\nu_{j}}$ 's are those zeros of $\rho_{m}$ which correspond to the zeros of $\tilde{g}_{m}$ lying outside of the unit disk by (2.6), $l_{j}$ is the multiplicity of the zero $\alpha_{\nu_{j}}$, and the $\mu_{k, j}$ 's are certain real numbers.

We now give the announced explicit expression for the kernel function $K_{n}\left(z, w_{\alpha, \beta}\right),|\alpha|=|\beta|=\frac{1}{2}$.

Theorem. Let $\rho_{m}$ be given by (2.4). Then we have for $y \in \mathbf{C} \backslash[-1,1]$, on writing $y=\frac{1}{2}\left(z+z^{-1}\right)$ with $|z|<1$, i.e., $z=y-\sqrt{y^{2}-1}$, that

$$
\begin{gathered}
c K_{n}\left(y, w\left(\cdot, \rho_{m}\right)\right)=\frac{4 z^{2 n+1}}{\left(1-z^{2}\right) g_{m}^{*}(z)\left\{z^{2 n-m} g_{m}(z)+g_{m}^{*}(z)\right\}} \quad \text { for } 2 n>m, \\
c K_{n}\left(y,\left(1_{(-)}^{+} x\right) w\left(\cdot, \rho_{m}\right)\right)=\frac{2 z^{2 n+1}\left(z_{(-)}^{+} 1\right)}{\left(1_{(+)}^{-} z\right) g_{m}^{*}(z)\left\{z^{2 n+1-m} g_{m}(z){ }_{(-)}^{+} g_{m}^{*}(z)\right\}} \\
\quad \text { for } 2 n+1>m, \\
c K_{n}\left(y,\left(1-x^{2}\right) w\left(\cdot, \rho_{m}\right)\right)=\frac{z^{2 n+1}\left(z^{2}-1\right) \quad}{g_{m}^{*}(z)\left\{z^{2 n+2-m} g_{m}(z)-g_{m}^{*}(z)\right\}} \\
\text { for } 2 n+2>m,
\end{gathered}
$$

where $g_{m}^{*}(z)=z^{m} g_{m}\left(\frac{1}{z}\right)$.

Proof. Let $R$ and $S$ be monic polynomials of degree at most two such that

$$
R(y) S(y)=y^{2}-1,
$$

and let us put for abbreviation

$$
P_{n}(x):=P_{n}\left(x, R w\left(\cdot, \rho_{m}\right)\right) \text { and } \tilde{P}_{n}(x):=P_{n}\left(x, S w\left(\cdot, \rho_{m}\right)\right) .
$$

Using the simple fact that for $k \in \mathbf{Z}$ and $\varphi \in[0,2 \pi]$

$$
\left[\operatorname{Re}\left\{e^{i k \varphi} g_{m}\left(e^{i \varphi}\right)\right\}\right]^{2}+\left[\operatorname{Im}\left\{e^{i k \varphi} g_{m}\left(e^{i \varphi}\right)\right\}\right]^{2}=\left|g_{m}\left(e^{i \varphi}\right)\right|^{2},
$$

we get, using (2.7) and (2.4), that with $l=n+\partial R-1$, where $\partial R$ denotes the exact degree of $R$,

$$
R P_{n}^{2}-S \widetilde{P}_{l}^{2}=k_{n} \rho_{m}
$$


where

$$
k_{n}= \begin{cases}2^{-2 n+2} / c & \text { for } R(x) \equiv 1, \\ -2^{-2 n} / c & \text { for } R(x) \equiv x^{2}-1, \\ +{ }_{(-)} 2^{-2 n+1} / c & \text { for } R(x) \equiv x+1(x-1) .\end{cases}
$$

Furthermore, it follows from Theorem $3(a)$ of our paper [9] that for $2 n \geq$ $m+1-\partial R$

$$
R P_{n}^{2}-S\left(Y P_{n}+\rho_{m} P_{n-1}^{(1)}\right)^{2}=d_{n} \rho_{m},
$$

where $P_{n-1}^{(1)}$ denotes the associated polynomial of $P_{n}$, i.e.,

$$
P_{n-1}^{(1)}(y)=\int_{-1}^{1} \frac{P_{n}(y)-P_{n}(x)}{y-x} R(x) w\left(x, \rho_{m}\right) d x,
$$

and $Y \in \mathbf{P}_{\mu}, \mu=\max \{m-1, \partial R-1\}$, is uniquely determined by the conditions that at each zero $\alpha_{\nu}$ of $\rho_{m}(x)=\tilde{c} \prod_{\nu=1}^{m^{*}}\left(\alpha_{\nu}-x\right)^{m_{\nu}}$, where $\tilde{c} \in \mathbf{R}$ and $m_{\nu}$ is the multiplicity of the zero $\alpha_{\nu}$,

$$
Y^{(j)}\left(\alpha_{\nu}\right)=\left(R / \sqrt{y^{2}-1}\right)^{(j)}\left(\alpha_{\nu}\right) \text { for } j=0, \ldots, m_{\nu}-1,
$$

and that for $y \rightarrow \infty$

$$
\frac{\left(R / \sqrt{y^{2}-1}-Y\right)(y)}{\rho_{m}(y)}=O\left(y^{-1}\right)
$$

furthermore,

$$
d_{n}=2 \int_{-1}^{+1} P_{n}^{2}(x) R(x) w\left(x, \rho_{m}\right) d x .
$$

(We note that in the definition of $1 / h$ in $\left[9\right.$, p. 461] $(-1)^{l-k} / \sqrt{-H}$ is to be replaced by $(-1)^{l-k} / \pi \sqrt{-H}$.) It now follows from [10, (2.6.5)] that the leading coefficient of the orthonormal polynomial of degree $n$ with respect to $R w\left(\cdot, \rho_{m}\right)$ is equal to $\sqrt{2 / k_{n}}$ for $2 n \geq m+1-\partial R$, which implies that $d_{n}=k_{n}$ and thus, in view of (2.9) and (2.10),

$$
\pm \widetilde{P}_{l}=Y P_{n}+\rho_{m} P_{n-1}^{(1)} \quad \text { for } 2 n \geq m+1-\partial R .
$$

For a function $f$ defined on $\mathbf{C} \backslash[-1,1]$ and for $x \in(-1,1)$ we write, provided the limits involved exist,

$$
f^{(\stackrel{+}{-})}(x):=\lim _{\substack{z \rightarrow x \\ z \in \mathbf{C}^{(-)}}} f(z)
$$

where $\mathbf{C}^{(\stackrel{+}{-})}:=\left\{z \in \mathbf{C}: \operatorname{Im} z_{(<)}^{>} 0\right\}$. Observing that by $(2.11)$ and $(2.12)$

$$
\Phi(y):=\frac{\left(R / \sqrt{y^{2}-1}-Y\right)(y)}{\rho_{m}(y)}
$$

is analytic on $C \backslash[-1,1]$ and vanishes at infinity, and that the boundary values $\Phi^{ \pm}(x), x \in(-1,1)$, from the upper (lower) half plane satisfy the relation

$$
\Phi^{+}(x)-\Phi^{-}(x)=\frac{2}{i} \frac{R(x)}{\rho_{m}(x) \sqrt{1-x^{2}}} \text { for } x \in(-1,1),
$$


where we have used the fact that

$$
\left(\sqrt{y^{2}-1}\right)^{+}(x)=i \sqrt{1-x^{2}}=-\left(\sqrt{y^{2}-1}\right)^{-}(x),
$$

we get by the Sochozki-Plemelj formula (see, e.g., [7])

$$
\begin{aligned}
\Phi(y) & =\frac{1}{\pi} \int_{-1}^{+1} \frac{1}{y-x} \frac{R(x)}{\rho_{m}(x) \sqrt{1-x^{2}}} d x \\
& =Q_{0}\left(y, R w\left(\cdot, \rho_{m}\right)\right) \text { for } y \in \mathbf{C} \backslash[-1,1] .
\end{aligned}
$$

Recalling the well-known fact (see, e.g., $[10, \S 3.5])$ that for sufficiently large $|y|$

$$
\frac{P_{n-1}^{(1)}(y)}{P_{n}(y)}=Q_{0}\left(y, R w\left(\cdot, \rho_{m}\right)\right)+O\left(y^{-(2 n+1)}\right) \text {, }
$$

we get, using (2.16) and (2.15), that

$$
\left(Y P_{n}+\rho_{m} P_{n-1}^{(1)}\right)(y)=P_{n}(y) R(y) / \sqrt{y^{2}-1}+O\left(y^{-(n+1)+m}\right)
$$

and thus, since for $2 n \geq m+1-\partial R$

$$
\lim _{y \rightarrow \infty} y^{-(n+\partial R-1)}\left\{P_{n}(y) R(y) / \sqrt{y^{2}-1}+O\left(y^{-(n+1)+m}\right)\right\}=1,
$$

the polynomial $Y P_{n}+\rho_{m} P_{n-1}^{(1)}$, which by (2.14) is of exact degree $n+\partial R-1$, has leading coefficient one. Hence, the plus sign holds in (2.14). Thus, the $n$th function of the second kind is of the form

$$
\begin{aligned}
Q_{n}\left(y, R w\left(\cdot, \rho_{m}\right)\right)=-P_{n-1}^{(1)}(y)+P_{n}(y) Q_{0}(y) \\
\quad=\frac{\sqrt{R(y)} P_{n}(y)-\sqrt{S(y)} \widetilde{P}_{l}(y)}{\sqrt{S(y)} \rho_{m}(y)}=\frac{k_{n}}{\left(\sqrt{y^{2}-1} P_{n}+S \widetilde{P}_{l}\right)(y)},
\end{aligned}
$$

where the second equality follows with the help of (2.16), (2.15), and (2.14), and the third equality with the help of (2.9). Now the following equalities hold on the circumference $|z|=1$ :

$$
\begin{gathered}
2^{n} P_{n}\left(\frac{1}{2}\left(z+z^{-1}\right), w\left(\cdot, \rho_{m}\right)\right)=z^{-n}\left(z^{2 n-m} g_{m}(z)+g_{m}^{*}(z)\right) \\
2^{n-1} P_{n-1}\left(\frac{1}{2}\left(z+z^{-1}\right),\left(1-x^{2}\right) w\left(\cdot, \rho_{m}\right)\right) \\
=\frac{z^{-n}\left(z^{2 n-m} g_{m}(z)-g_{m}^{*}(z)\right)}{z-z^{-1}}, \\
2^{n} P_{n}\left(\frac{1}{2}\left(z+z^{-1}\right),\left(1_{(-)}^{+} x\right) w\left(\cdot, \rho_{m}\right)\right) \\
=\frac{z^{-n}\left(z^{2 n+1-m} g_{m}(z)_{(-)}^{+} g_{m}^{*}(z)\right)}{z_{(-)}^{+} 1^{+}} .
\end{gathered}
$$

Since all functions appearing in (2.18) are analytic in the domain $\mathbf{C} \backslash\{0\}$, it follows that in (2.18) equality holds also on $\mathbf{C} \backslash\{0\}$. Hence, we get from (2.17) 
and (2.18) that for $y \in \mathbf{C} \backslash[-1,1]$, on writing $y=\frac{1}{2}\left(z+z^{-1}\right)$ with $|z|<1$,

$$
\begin{aligned}
Q_{n}\left(y, w\left(\cdot, \rho_{m}\right)\right) & =\frac{2^{-n+2}}{c} \frac{z^{n+1}}{\left(1-z^{2}\right) g_{m}^{*}(z)}, \\
Q_{n}\left(y,\left(1-x^{2}\right) w\left(\cdot, \rho_{m}\right)\right) & =\frac{2^{-n}}{c} \frac{z^{n+1}}{g_{m}^{*}(z)}, \\
Q_{n}\left(y,\left(1_{(+)}^{-} x\right) w\left(\cdot, \rho_{m}\right)\right) & =\frac{2^{-n+1}}{c} \frac{z^{n+1}}{\left(1_{(-)}^{+} z\right) g_{m}^{*}(z)},
\end{aligned}
$$

where we have used the fact that $\sqrt{y^{2}-1}=\left(z^{-1}-z\right) / 2$. Relation (2.19) in conjunction with (2.18) and (1.4) gives the assertion.

In the remark below we state sufficient conditions on the weight function $w_{\alpha, \beta}\left(x, \rho_{m}\right)$, defined in (1.13), such that (1.10), resp. (1.11), is fulfilled. Since the product $w_{1}(x) w_{2}(x)$ of two weight functions $w_{1}, w_{2}$ satisfies condition (1.10), resp. (1.11) if $w_{1}$ and $w_{2}$ satisfy (1.10), resp. (1.11), we consider the behavior of $w\left(x, \rho_{m}\right) / w\left(-x, \rho_{m}\right)$ for $m \in\{1,2\}$ only.

Remark. The ratio $w(x, \rho) / w(-x, \rho)$ is nondecreasing (nonincreasing) on $(-1,1)$ if

$$
\rho(x)= \begin{cases}\stackrel{+}{(-)}(\alpha-x), & \alpha \in(1, \infty)((-\infty,-1)), \\ (\alpha-x)(x-\beta), & \alpha \in(1, \infty), \beta \in(-\infty,-1), \text { and }-\beta \stackrel{(\leq)}{\geq}^{\geq} \alpha, \\ (x-\alpha)^{2}+\beta^{2}, & \alpha \in \mathbf{R}_{(-)}^{+}, \beta \in \mathbf{R}, \text { and } \alpha^{2}+\beta^{2} \geq 1,\end{cases}
$$

where the expressions in parentheses refer to the case of nonincreasing ratio.

Setting in the preceding theorem

$$
g_{1}(z)=z+\tilde{a}, \quad \tilde{a} \in(-1,1), \quad \text { i.e., }\left|g_{1}\left(e^{i \varphi}\right)\right|^{2}=1+\tilde{a}^{2}+2 \tilde{a} x,
$$

resp. for $b>0$

$$
g_{2}(z)=z^{2}+(1+2 b)^{-1}, \quad \text { i.e., }\left|g_{2}\left(e^{i \varphi}\right)\right|^{2}=4\left(b^{2}+(1+2 b) x^{2}\right) /(2 b+1)^{2},
$$

where $x=\cos \varphi$, we obtain the results of Kumar [5,6] concerning the functions of the second kind, and the results of Akrivis [1] on the norm of the error functional $R_{n}\left(\cdot, w_{\alpha, \beta}\left(\cdot,\left|g_{j}\left(e^{i \varphi}\right)\right|^{2}\right)\right), j=1,2$. If we put

$$
g_{2}(z)=z^{2}+\frac{2 \delta}{\beta} z+\left(1-\frac{2 \alpha}{\beta}\right)
$$

with $0<\alpha<\beta, \beta \neq 2 \alpha$, and $|\delta|<\beta-\alpha$, which gives

$$
\frac{\beta^{2}}{4}\left|g_{2}\left(e^{i \varphi}\right)\right|^{2}=\beta(\beta-2 \alpha) x^{2}+2 \delta(\beta-\alpha) x+\alpha^{2}+\delta^{2},
$$

we obtain the results of Notaris [8] on the norm of the error functional, using his conditions $\left(2.3_{1}\right)-\left(2.4_{2}\right)$ on the parameters $\alpha, \beta, \gamma, \delta$ under which the function $w\left(x,\left|g_{2}\left(e^{i \varphi}\right)\right|^{2}\right) / w\left(-x,\left|g_{2}\left(e^{i \varphi}\right)\right|^{2}\right)$ is strictly increasing, resp. strictly decreasing.

\section{ACKNOWLEDGMENT}

I would like to thank the referee for a careful reading of the manuscript and for his valuable comments. 


\section{BibLIOGRAPHY}

1. G. Akrivis, The error norm of certain Gaussian quadrature formulae, Math. Comp. 45 (1985), 513-519.

2. W. Gautschi, On Padé approximants associated with Hamburger series, Calcolo 20 (1983), 111-127.

3. W. Gautschi and R. S. Varga, Error bounds for Gaussian quadrature of analytic functions, SIAM J. Numer. Anal. 20 (1983), 1170-1186.

4. G. Hämmerlin, Fehlerabschätzungen bei numerischer Integration nach Gauss, Methoden und Verfahren der Mathematischen Physik, vol. 6 (B. Brosowski and E. Martensen, eds.), Bibliographisches Institut, Mannheim, Wien, Zürich, 1972, pp. 153-163.

5. R. Kumar, A class of quadrature formulas, Math. Comp. 28 (1974), 769-778.

6. __ Certain Gaussian quadratures, J. Inst. Math. Appl. 14 (1974), 175-182.

7. N. I. Muschelischwili, Singuläre Integralgleichungen, Akademie Verlag, Berlin, 1965.

8. S. E. Notaris, The error norm of Gaussian quadrature formulae for weight functions of Bernstein-Szegö type, Numer. Math. 57 (1990), 271-283.

9. F. Peherstorfer, On Bernstein-Szegö orthogonal polynomials on several intervals, SIAM J. Math. Anal. 21 (1990), 461-482.

10. G. Szegö, Orthogonal polynomials, 3rd ed., Amer. Math. Soc. Colloq. Publ., vol. 23, Amer. Math. Soc., Providence, RI, 1967.

Institut für Mathematik, Johannes KePler Universität Linz, A-4040 LinZ, AUStria 